\title{
An Alternative View of Uncertainty in Bilateral Bargaining Models with Incomplete Information
}

\author{
Javier González and Vitaliy V. Kalashnikov
}

\begin{abstract}
This paper seeks to analyze the implications of relaxing the assumption of rationality by using incomplete information. Specifically, we want to analyze the implications on the uniqueness and existence of equilibrium when individuals form expectations with different distributions of all possible states. This is done by postulating an alternative view of uncertainty in decision making in economic interactions. Thus an alternative model of bilateral bargaining with incomplete information is formulated.
\end{abstract}

\section{Introduction}

Over the last 60 years game theory has become an important tool in economic modeling of strategic decision making in interactive situations. Particularly, game theoretic models have provided a solid framework for the study of strategic interaction previously lacking in the field of economics. The introduction of a higher level of mathematical precision and sophistication has come, however, at the expense of accuracy of its predictions and consistency with observed behavior. That is, fundamental assumptions have become more restrictive in the development of oversimplified models trying to predict behavior. One such fundamental assumption in game theory is rational behavior by the decision-maker, or rationality [8]. Within the context of game theory rationality refers to the ability of an individual to consistently make decisions that maximize that individual's expected payoff given varying levels of knowledge. In the absence of 'uncertainty' rationality implies an individual's full knowledge (i.e., complete information) of the structure of the game, cognitive ability to weigh different choices and deliberately make optimal decisions. In the presence of uncertainty about the game structure (i.e., incomplete information) rationality

Javier González · Vitaliy V. Kalashnikov

Graduate School of Economics, UANL, Av. Lázaro Cárdenas S/N,

Monterrey, N.L., Mexico 64890

e-mail:javier.gonzalez.g@gmail.com,kalashnikov_de@yahoo.de

J. Watada et al. (Eds.): Intelligent Decision Technologies, Vol. 1, SIST 15, pp. 109-118. springerlink.com

(c) Springer-Verlag Berlin Heidelberg 2012 
implies an individual's ability to choose actions that maximize expected payoffs given full knowledge of all possible games and their distribution. It is the assumption of rational behavior when players are uncertain about the probability distribution their opponents assign to the different 'states' of the game that we seek to relax. Then implications of relaxing said assumption on the existence and uniqueness of a Nash equilibrium are analyzed. Our goal is to introduce this alternative view of uncertainty in decision making in economic interactions with the purpose of formulating an alternative model of decision making in bilateral bargaining games with incomplete information.

Under the assumption of (super) rationality in the absence of uncertainty, in 1950 John Nash [6] developed an axiomatic framework to form an equilibrium solution to the bilateral bargaining problem. While valid under specific complete information conditions, Nash's bargaing model appears to be inconsistent with observed behavior when players are faced with incomplete information. Looking to overcome Nash's model inconsistencies, Jonh Harsanyi [2] formulated a model that accounts for players with less-than-complete information about the structure of the game. In the presence of (unmeasurable) uncertainty of the structure of bilateral bargaining game, Harsanyi assumes players will form a commonly known set of possible game structures and a corresponding probability distribution (i.e., imperfect information). That is, players will transform a game of unknown possible actions and outcomes into a game where the distribution of all possible actions and outcomes is commonly known. Thus, players can form an expectation of a set of possible actions that satisfies the conditions for a Nash Equilibrium.

While Harsanyi's model holds under common knowledge of different sets of actions and outcomes with a common probability distribution, it appears to be inconsistent with observed behavior once players' are endowed with different probability distributions. The objective of the present study is to analyze the implications of introducing unmeasurable uncertainty [4] (Knightian Uncertainty) into the existing framework of bilateral bargaining models. Specifically, we model bilateral bargaining where players assign different probabilities over all possible actions and outcomes leading to a disagreement that is inconsistent with theoretical predictions. Thus providing the foundation for a model of bilateral bargaining with incomplete information under unmeasurable uncertainty.

We begin our task in section 2 by describing the general bilateral bargaining model with incomplete information, and assuming rational behavior. We assume each player has full knowledge of all the possible states of the game, as well as the probability of each state. Thus we rewrite the game of incomplete information as a game of imperfect information. Next in section 3 we relax the assumption of rationality, introducing uncertainty of the probability a player assigns over all the possible states of the game. Specifically, we allow each player to have a different (subjective) probability distribution over all possible states. The implications of this are examined conceptually, then illustrated by an example. Finally, conclusions are provided in section 4 . 


\section{Measurable Uncertainty and Mutual Consistency of Beliefs in Bilateral Bargaining}

Under Nash's axiomatic model [6] with complete information for a bilateral bargaining game with a solution of the form

$$
f^{N}(S, d)=\underset{\left(d_{1}, d_{2}\right) \leq\left(x_{1}, x_{2}\right) \in S}{\operatorname{argmax}}\left(x_{1}-d_{1}\right)\left(x_{2}-d_{2}\right)
$$

subject to a bargaining set

$$
S(d)=\left\{\left(x_{1}, x_{2}\right) \in S \mid x_{1}+x_{2} \leq c, x_{i} \geq d_{i}, S \subset R^{2}\right\}
$$

where $x_{i}$ is the payoff demand for player $i$ within the agreement convex space $S$. This set is bounded below by a disagreement payoff $d_{i}$ and it is assumed that both players have complete knowledge of each other's possible actions. To take account of incomplete information in games, Harsanyi [2] proposed a model that transforms a game of incomplete information into a game of imperfect information. Under the framework of incomplete information with measurable uncertainty proposed by Harsanyi, the information of the different sets of actions and payoffs are associated with a distribution of 'player types' with given probabilities. Thus, in the context of a bilateral bargaining game with a normal form for each player $i=1,2$ with an arbitrary number of types $k$, a vector of types for player $i$ is represented by $c_{i}=$ $\left\{c_{i}^{1}, c_{i}^{2}, \ldots, c_{i}^{k}\right\}$ which is used to normalize strategies under complete information for every possible $k$ type such that $s_{i} \mapsto s_{i}^{*}=s_{i}^{*}\left(c_{i}\right)=\left\{s_{i}^{*}\left(c_{i}^{1}\right), s_{i}^{*}\left(c_{i}^{2}\right), \ldots, s_{i}^{*}\left(c_{i}^{k}\right)\right\}$, and each player $i$ has a vector of subjective probabilities (probability distribution) of all the possible types for his opponent given his own, such that $\bar{P}_{i}\left(c_{j}\right)=P_{i}\left(c_{j} \mid c_{i}\right)$ for $j=1,2, j \neq i$. Let $S_{i}$ be the set of all possible strategies for all $k$ types for player $i=1,2, C_{i}=\left\{c_{i}\right\}$ the set of all vector types. Let the game of incomplete information be defined as $G=\left\{S_{1}, S_{2}, C_{1}, C_{2}, V_{1}, V_{2}, P_{1}, P_{2}\right\}$ where $V_{i}:\left[S_{1} \times S_{2} \times C_{1} \times C_{2}\right] \rightarrow R$ represents player $i$ 's payoff function.

Under the central assumption that the vectors of subjective probabilities of both players are equal, i.e., $\bar{P}_{1}\left(c_{2}\right)=\bar{P}_{2}\left(c_{1}\right): P_{i}\left(c_{j} \mid c_{i}\right)=P^{*}\left(c_{j} \mid c_{i}\right)$ for $i, j \in\{1,2\}, i \neq j$, Harsanyi's model claims that it is possible to derive a commonly known objective probability distribution such that

$P^{*}\left(c_{j} \mid c_{i}\right)=\frac{P^{*}\left(c_{i}, c_{j}\right)}{\int_{C_{j}} d_{\left(c_{j}\right)} P^{*}\left(c_{i}, c_{j}\right)} \Rightarrow P^{*}\left(c_{i}, c_{j}\right)=P^{*}(c)=P_{i}\left(c_{i}, c_{j}\right) \int_{C_{j}} d_{\left(c_{j}\right)} P^{*}\left(c_{i}, c_{j}\right)$

Using player $i$ 's normalized strategies, $s_{i}^{*}\left(c_{i}\right)$, normalized payoffs are derived, $x_{i}=$ $V_{i}\left(s_{1}^{*}\left(c_{1}\right), s_{2}^{*}\left(c_{2}\right), c_{1}, c_{2}\right)$, which in turn provides a weighted sum of the normalized strategies that are used to represent player $i$ 's expected payoffs in the normal form of the game:

$$
E x_{i}=W_{i}\left(s_{1}^{*}, s_{2}^{*}\right)=\int_{C} V_{i}\left(s_{1}^{*}, s_{2}^{*}\right) d_{(c)} P^{*}(c)
$$


which can be modified to account for player $i$ 's expected payoffs given his own type

$$
E\left(x_{i} \mid c_{i}\right)=Z_{i}\left(s_{1}^{*}, s_{2}^{*} \mid c_{i}\right)=\int_{C_{j}} V_{i}\left(s_{1}^{*}, s_{2}^{*}, c_{1}, c_{2}\right) d_{\left(c_{j}\right)} P^{*}\left(c_{j} \mid c_{i}\right)
$$

Assuming that for player $i$ 's normalized strategy, $s_{i}^{*}$, a specific vector of types, $c_{i}^{0}$, there exists a corresponding optimal ordinary strategy $s_{i}=s_{i}^{*}\left(c_{i}\right)$ that maximizes the expected payoff, $E\left(x_{i} \mid c_{i}^{0}\right)=Z_{i}\left(s_{1}^{*}, s_{2}^{*} \mid c_{i}^{0}\right)$ such that $s_{i}^{*}$ is the best response for $c_{i}^{0}$ given his opponent's strategy $s_{j}^{*}$, then for $s_{i}^{*}$ being a uniformly best response for $c_{i}$ except for a small subset of types $c_{i}^{*}$ - with probability nearly zero $-\left\{s_{i}^{*} \cup s_{j}^{*}\right\}, i, j \in\{1,2\}, i \neq j$ gives us an equilibrium point. This implies that in order to have an equilibrium for the complete and imperfect information $G^{*}=\left\{s_{1}, s_{2}, c_{1}, c_{2}, V_{1}, V_{2}, P^{*}\right\}$, which is analog to the game of incomplete information $G$, it is sufficient and necessary for the set of normalized strategies $s^{*}=\left(s_{1}^{*}, s_{2}^{*}\right)$ to be a Nash Equilibrium.

Holding the assumption of mutually consistent subjective probability distributions, $P_{i}^{*}=P^{*}(c), i=1,2$, with know player type vectors $\left\{c_{1}, c_{2}\right\}$, a generalized bilateral bargaining model is elaborated subject to a set of all possible agreements defined as

$$
X(d)=\left\{\left(x_{1}\left(c_{1}\right), x_{2}\left(c_{2}\right)\right) \mid x_{1}\left(c_{1}\right)+x_{2}\left(c_{2}\right) \leq z, x_{i}\left(c_{i}\right) \geq d_{i}\right\}
$$

where $X \subset R^{2}, d_{i} \geq 0$, for all possible combinations of known types given by the Cartesian product $C_{1} \times C_{2}$. Furthermore, given that the payoff for each player $i$, $x_{i}\left(c_{i}\right)$ is the weighted sum of all possible normalized strategies given $c_{i}$, the set of all possible agreements $X(d)$ is the weighted sum of all the possible $(k \times k)$ bargaining subsets of payoff vectors for all specific types, $c_{1}^{l} \in C_{1}, c_{2}^{m} \in C_{2}$, for all $l, m \in\{1,2, \ldots, k\}:$

$$
X_{l m}(d)=\left\{\left(x_{1}\left(c_{1}^{l}\right), x_{2}\left(c_{2}^{m}\right)\right) \mid x_{1}^{l}+x_{2}^{m} \leq z, x_{i}^{\bullet} \geq d_{i}^{\bullet}\right\}
$$

where each vector pair type specific bargaining subset $X_{l m}(d)$ adheres to Nash formulation and satisfies its conditions, such that there exists a rectangle $T_{l m}$ formed by the separating hyperplane that bounds $X_{l m}(d)$ given a hyperbolic curve formed by the objective function of the bargaining solution $H=\left(x_{1}-d_{1}\right)\left(x_{2}-d_{2}\right)$. Thus, the product of all type pair specific bargaining sets, which is a convex combination of every set represented by (3), forms a set of all possible bargaining agreements such that for $C_{1} \times C_{2}$

$$
X(d)=\prod_{(l, m) \in(K, K)} X_{l m}(d), \quad K=\{1,2, \ldots, k\}
$$

where the $\prod X_{l m}$ for $(l, m) \in(K, K)$ provides a weighted sum given the distribution of possible types for the common probability matrix $P^{*}$. Using Nash's axiom of 
independence of irrelevant alternatives, which states that the optimal point $x^{*} \in X_{l m}$ is also the optimal point in $T_{l m}$, let the weighted space of all bounding $T_{l m}$ rectangles that forms a convex hull in $R_{+}^{2}$ be

$$
T=\prod_{(l, m) \in(K, K)} T_{l m}, \quad K=\{1,2, \ldots, k\}
$$

satisfying convexity conditions necessary for existence and uniqueness of a Nash Equilibrium.

\section{Unmeasurable Uncertainty and Inconsistency of Beliefs in Bilateral Bargaining}

While Harsanyi's model with imperfect information holds under mutual consistency among players' probability distributions, Harsanyi [3] among others [5] recognized the possible failure of this model upon relaxing the assumption of mutual consistency. Specifically, Harsanyi acknowledge that by the nature of subjective probabilities even when two individuals have the same information and the same level of intelligence, thy may still assign different subjective probabilities to the same events. By relaxing the assumption of mutual consistency of the probability distributions among players, $P_{1}\left(c_{1}, c_{2}\right) \neq P_{2}\left(c_{1}, c_{2}\right)$. Thus, the ability to derive a common probability distribution $P^{*}$ to construct an analogous game of complete but imperfect information $G^{*}$ is no longer valid.

It is assumed that the type vectors, $c_{i}=\left(c_{i}^{1}, c_{i}^{2}, \ldots, c_{i}^{k}\right)$, are commonly known by both players while having different distributions of all the possible combinations of type vector pairs that span the space of the cartesian product $C_{1} \times C_{2}$. Then for each player $i$ according to their type vector, $c_{i}$, and the corresponding set of normalized strategies $s_{i}=s_{i}^{*}\left(c_{i}\right)=\left\{s_{i}^{*}\left(c_{i}^{1}\right), s_{i}^{*}\left(c_{i}^{2}\right), \ldots, s_{i}^{*}\left(c_{i}^{k}\right)\right\}$, we can define the game of incomplete information $G=\left\{S_{1}, S_{2}, C_{1}, C_{2}, V_{1}, V_{2}, P_{1}, P_{2}\right\}$ - where once again $V_{i}$ : $\left[S_{1} \times S_{2} \times C_{1} \times C_{2}\right] \rightarrow R$ represents the payoffs for player $i$ in the normal for of the game.

Given the relaxation of mutual consistency of distributions, $P_{1} \neq P^{*} \neq P_{2}$, each player $i$ will assign different normalized payoffs to both players. The difference in normalized payoffs will produce a different weighted sum for each player. Thus, the normalized payoffs according to player $i$ 's distribution such that $x_{i}^{i}=$ $V_{i}\left(s_{1}^{i *}\left(c_{1}\right), s_{2}^{i *}\left(c_{2}\right), c_{1}, c_{2}\right)$ and $x_{j}^{i}=V_{j}\left(s_{1}^{i *}\left(c_{1}\right), s_{2}^{i *}\left(c_{2}\right), c_{1}, c_{2}\right)$ for $i, j \in\{1,2\}, i \neq j$ will produce different agreement vectors than those produced according to player $j$ 's, .ie., $\left(x_{1}^{i}, x_{2}^{i}\right) \neq\left(x_{1}^{j}, x_{2}^{j}\right)$. Specifically, the expected payoffs in the normal form of the game will differ among players given that each player's weighted sum will assign a different probability weighting over the space of all possible combinations of type vector pairs, such that 


$$
\begin{gathered}
E\left(x_{i}^{i} \mid c_{i}\right)=\int_{C_{j}} V_{i}\left(s_{i}^{i *}, s_{j}^{i *}, c_{i}, c_{j}\right) d_{\left(c_{j}\right)} P_{i}^{i}\left(c_{j} \mid c_{i}\right), E\left(x_{j}^{i} \mid c_{j}\right)= \\
=\int_{C_{i}} V_{j}\left(s_{i}^{i *}, s_{j}^{i *}, c_{i}, c_{j}\right) d_{\left(c_{i}\right)} P_{j}^{i}\left(c_{i} \mid c_{j}\right)
\end{gathered}
$$

for $i, j \in\{1,2\}, i \neq j$ where $P_{j}^{i}\left(c_{i} \mid c_{j}\right)$ is player $i$ 's estimate of the conditional probability player $j$ assigns $\hat{P}_{j}=P_{i}^{j}$ for $c_{i}$ given $c_{j}$. Thus, player $i$ uses a sum expected payoffs which assigns a different probability weight for each possible normalized strategy, $E\left(x_{j}^{j} \mid c_{j}\right) \neq E\left(x_{j}^{i} \mid c_{j}\right)$.

Let us suppose that both players wrongly assume that their subjective probability distributions are mutually consistent such that $P_{i}^{i}=\hat{P}_{i}$ and $P_{j}^{j}=\hat{P}_{j}$ for $i, j \in\{1,2\}, i \neq j$. Thus, each player $i$ will be trying to solve a different game of imperfect information $G_{i}^{*}=\left\{S_{1}^{i *}, S_{2}^{i *}, C_{1}, C_{2}, V_{1}, V_{2}, P^{i *}\right\}$ where $P^{i *}$ is what player $i$ perceives to be the commonly known probability distribution over all possible type vectors. For the generalized bargaining model defined in the previous section with known type vectors $\left\{c_{1}, c_{2}\right\}$ and mutually inconsistent (unknown) probability distributions, $P_{i} \neq P_{j} \rightarrow P^{i *} \neq P^{j *}$, the set of all possible agreements given the set of expected payoffs for both players according to player $i, x^{i}=\left(x_{i}^{i}, x_{j}^{i}\right)$ such that

$$
X^{i}(d)=\left\{\left(x_{1}^{i}\left(c_{1}\right), x_{2}^{i}\left(c_{2}\right)\right) \mid x_{1}^{i}\left(c_{1}\right)+x_{2}^{i}\left(c_{2}\right) \leq z, x_{i}^{i}\left(c_{i}\right) \geq d_{i}\right\}
$$

for $d_{i} \geq 0, i=1,2$ will be different than the set of all possible agreements given the set of expected payoffs for both players according to player $j, x^{j}=\left(x_{i}^{j}, x_{j}^{j}\right)$ such that for $x^{i} \neq x^{j} \longrightarrow X^{i}(d) \neq X^{j}(d)$, where both are sets are in $R^{2}$.

While the set of all possible $(k \times k)$ type vector pair combinations from the cartesian product of all type vectors for each player, $C_{1} \times C_{2}$, remain the same, given different probability weighting of all normalized strategies, any particular combination of type vector pair, $c_{1}^{l}, c_{2}^{m}$ for $l, m \in\{1,2, \ldots, k\}$ will produce different type vector specific bargaining sets, such that

$$
X_{l m}^{i}(d)=\left\{\left(x_{1}^{i}\left(c_{1}^{l}\right), x_{2}^{i}\left(c_{2}^{m}\right)\right) \mid x_{1}^{i}\left(c_{1}^{l}\right)+x_{2}^{i}\left(c_{2}^{m}\right) \leq z, x_{i}^{\bullet} \geq d_{i}^{\bullet}\right\}
$$

for $i=1,2 ; l, m \in\{1,2\}$, where (5) satisfies the conditions for Nash solution and it is bounded by a rectangle $T_{l m}^{i}$ formed by a separating hyperplane produced by the hyperbolic curve formed by the objective function in Nash's bargaining solution, $H=\left(x_{1}^{i}-d_{1}\right)\left(x_{2}^{i}-d_{2}\right)$ according what player $i$ perceives to be the vector of expected payoffs for both players, $x^{i}=\left(x_{1}^{i}, x_{2}^{i}\right)$. Thus, given different probability weighting for a specific vector type pair, $\left(c_{1}^{l}, c_{2}^{m}\right), X_{l m}^{i} \neq X_{l m}^{j}$, their respective weighted sums of all possible type vector specific agreements will form different sets of all possible convex combinations in $C_{1} \times C_{2}$ :

$$
X^{i}(d)=\prod_{(l, m) \in(K, K)} X_{l m}^{i}(d) \neq X^{j}(d)=\prod_{(l, m) \in(K, K)} X_{l m}^{j}(d)
$$


for $K=\{1,2, \ldots, k\}$. Thus, by Nash's axiom of Pareto optimality where $\left(x_{1}^{i *}\left(c_{1}^{l}\right), x_{2}^{i *}\left(c_{2}^{m}\right)\right) \in X_{l m}^{i} \rightarrow\left(x_{1}^{i *}\left(c_{1}^{l}\right), x_{2}^{i *}\left(c_{2}^{m}\right)\right) \in T_{l m}^{i}$, the weighted sum for each player $i$ of all possible type vector specific agreements will produce a convex hull for the affine combination of all bounding rectangles $T_{l m}^{i} \in R_{+}^{2}$, where $T_{l m}^{i} \neq T_{l m}^{j}$ such that

$$
T^{i}=\prod_{(l, m) \in(K, K)} T_{l m}^{i} \neq T^{j}=\prod_{(l, m) \in(K, K)} T_{l m}^{j}
$$

for $i, j \in\{1,2\}, l, m \in\{1,2\}, i \neq j, l \neq m$. While both convex hulls formed by $T^{i}$ and $T^{j}$ satisfy the convexity conditions for the existence of an equilibrium, the expected equilibrium payoffs formed by both players will differ. Thus, leading to a bargaining process whereby neither Pareto nor Nash equilibria can be reached - aside from the disagreement point. That is, the weights assigned to their normalized strategies will lead to different expectations as to what the payoff demands should be according to each player's type. Thus, the affine combination of their demands could result in payoff demand vectors outside the space formed by the union of both spaces of all possible agreements. This breaks the convexity (preserving) condition necessary for the existence of an equilibrium, which leads to the inability of players reaching a bargaining solution.

\subsection{Example of a Bilateral Bargaining Game with Incomplete Information and Inconsistency of Probability Distributions}

Let us suppose two players are playing a game of dividing $\$ 100$ where the privately known payoff demand of player $i$ is $y_{i}^{*}$ for $i=1,2$. If the sum of players' payoff demands do not exceed the given amount, i.e., $y_{1}^{*}+y_{2}^{*} \leq 100$, each player receives the payoff amount demanded, $y_{i}=y_{i}^{*}$. Otherwise, both players receive 0 . Given the set of pure payoff demands and the set of types for each player, $c_{i} \in\left\{a_{1}, a_{2}\right\}$, the set of expected payoffs for player $i$ are defined by

$$
x_{i}=\frac{1}{2} y_{i} \times \mathbf{1}\left(c_{i}=a_{1}\right)+y_{i} \times \mathbf{1}\left(c_{i}=a_{2}\right)
$$

where $\mathbf{1}(\cdot)$ is an indicator function and each player assigns a different probability to each possible type vector combination given by

$$
C_{1} \times C_{2}=\left\{\left(a_{1}, a_{1}\right),\left(a_{1}, a_{2}\right),\left(a_{2}, a_{1}\right),\left(a_{2}, a_{2}\right)\right\} .
$$

Let us assume that both players assign a negative correlation of types such that $\operatorname{Pr}\left(c_{j}=a_{m} \mid c_{i}=a_{l}\right)=1$ where $i, j \in\{1,2\}, l, m \in\{1,2\}, i \neq j, l \neq m$. If player 1 assigns an equal probability of having a pair of type vectors $\left(c_{1}, c_{2}\right)=\left(a_{1}, a_{2}\right)$ or $\left(c_{1}, c_{2}\right)=\left(a_{2}, a_{1}\right)$ such that $p_{12}^{1}=p_{21}^{1}=\frac{1}{2}$, then the vector of expected payoffs according to player 1 is given by

$$
x^{1}=\left(x_{1}^{1}, x_{2}^{1}\right)=\frac{1}{2} \cdot x^{1 *}+\frac{1}{2} \cdot x^{1 * *}
$$


where $x^{1 *}$ represents the possible payoff vectors given the pair of type vectors $\left(c_{1}, c_{2}\right)=\left(a_{1}, a_{2}\right)$ and $x^{1 * *}$ represents the possible payoff vectors given the pair of type vectors $\left(c_{1}, c_{2}\right)=\left(a_{2}, a_{1}\right)$. Thus, player 1 will formulate a weighted sum of possible payoff vectors for each player such that

$$
\begin{aligned}
& x_{1}^{1}=\frac{1}{2} \cdot\left(50-\frac{1}{2} x_{2}\right)+\frac{1}{2}\left(100-2 x_{2}\right) \\
& x_{2}^{1}=\frac{1}{2} \cdot\left(100-2 x_{1}\right)+\frac{1}{2} \cdot\left(50-\frac{1}{2} x_{1}\right)
\end{aligned}
$$

and form a space of all possible expected payoffs in the following manner.

- First, under the cooperative framework where player 1 receives the full amount $x_{1}=100$ while player 2 receives nothing $x_{2}=0$ when $\left(c_{1}, c_{2}\right)=\left(a_{2}, a_{1}\right)$ and player 1 receives nothing $x_{1}=0$ while player 2 receives the full amount $x_{2}=100$ with an equal probability of $\frac{1}{2}$, the expected Pareto optimal equilibrium payoff according to player 1 is $\left(x_{1}^{1}, x_{2}^{1}\right)=(50,50)$.

- Then, under the non-cooperative framework where each player's expected payoff is formed by the weighted sum of the Nash equilibrium outcomes under each pair of type vectors, the expected Nash equilibrium payoff according to player 1 is $\left(x_{1}^{1}, x_{2}^{1}\right)=(37.5,37.5)$.

- Finally, when players agree that player 1 receives the upper bound payment while player 2 receives the lowest bound payment the expected payoff vector according to player 1 is $\left(x_{1}^{1}, x_{2}^{1}\right)=(75,0)$. When players agree that player 1 receives the lowest bound payment while player 2 receives the upper bound payment the expect payoff according to player 1 is $\left(x_{1}^{1}, x_{2}^{1}\right)=(0,75)$.

Thus, the weighted space of all possible affine combinations of expected payoffs according to player 1 is represented by the convex hull shown in the figure below and is denominated as $X^{1}$.

Let us now assume that player 2 assigns unequal probabilities to the different pairs of type vectors where $\left(c_{1}, c_{2}\right)=\left(a_{1}, a_{2}\right)$ has a probability $p_{12}^{2}=\frac{3}{4}$ while $\left(c_{1}, c_{2}\right)=\left(a_{2}, a_{1}\right)$ has a probability $p_{21}^{2}=\frac{1}{4}$. The the vector of expected payoffs according to player 2 is given by

$$
x^{2}=\left(x_{1}^{2}, x_{2}^{2}\right)=\frac{3}{4} \cdot x^{2 *}+\frac{1}{4} \cdot x^{2 * *}
$$

where $x^{2 *}$ represents the possible payoff vectors given the pair of type vectors $\left(c_{1}, c_{2}\right)=\left(a_{1}, a_{2}\right)$ and $x^{2 * *}$ represents the possible payoff vectors given the pair of type vectors $\left(c_{1}, c_{2}\right)=\left(a_{2}, a_{1}\right)$. Thus, player 2 will formulate a weighted sum of possible payoff vectors for each player such that

$$
\begin{aligned}
& x_{1}^{2}=\frac{3}{4} \cdot\left(50-\frac{1}{2} x_{2}\right)+\frac{1}{4} \cdot\left(100-2 x_{2}\right) \\
& x_{2}^{2}=\frac{3}{4} \cdot\left(100-2 x_{1}\right)+\frac{1}{4} \cdot\left(50-\frac{1}{2} x_{2}\right)
\end{aligned}
$$


and through a similar process but with different weights than player 1 , the resulting space of all possible expected payoffs is formed by: (1) the expected Pareto optimal equilibrium payoff according to player $2,\left(x_{1}^{2}, x_{2}^{2}\right)=(32.5,60)$; (2) the expected Nash equilibrium according to player 2, $\left(x_{1}^{2}, x_{2}^{2}\right)=(31.25,43.75)$; (3) the upper bound payment for player 1 and lower bound payment for player 2 , $\left(x_{1}^{2}, x_{2}^{2}\right)=(62.5,0)$, and the lower bound payment for player 1 and upper bound payment for player $2,\left(x_{1}^{2}, x_{2}^{2}\right)=(0,87.5)$. Thus, the weighted space of all possible affine combinations of expected payoffs according to player 2 is represented by the convex hull.

By having different probability distributions over the set of possible pairs of type vectors, $P_{1} \neq P_{2}$, each player generates different weighted sums of possible outcomes. Thus, each player has different space of possible solutions with different probability weights assigned to their normalized strategies. Players will formulate different sets of optimal best-response strategies under the erroneous belief that both players have the same (mutually reinforcing) probability distribution. So even when both players have common knowledge of the structure of the game and agree to cooperate, given different distributions of probabilities of the different states, the models based on the assumption of rationality with mutual consistency of probability distributions fail to account for instance of nonexistence or uniqueness of an equilibrium.

\section{Conclusions}

As it was show in the previous example, we have a very different structure as compared to Nash and Harsanyi's models. The difference is explained by different distributions of the players' strategies. As it has been shown in experimental studies [1] [10] where players are privately induced with different distributions of all possible outcomes, when opposing players with different expectations of equilibrium payoff demands try to reach an agreement theory predictions are no longer consistent with observed behavior [1]. Far from attempting to discard existing models of bilateral bargaining of complete but imperfect information [2], we offer an alternative view of uncertainty to explain inconsistencies with theoretical predictions. Specifically, we develop a framework that accounts for varying degrees of knowledge and mutual consistency of beliefs in bilateral bargaining. Our goal in this particular analysis is to put forward a conjecture that establishes a direct relationship between the degree of inconsistency in beliefs among individuals and their ability to reach an agreement. Thus, providing the foundation for building a model of bilateral bargaining with incomplete information under an unmeasurable uncertainty framework [4].

Acknowledgements. This research was supported by the National Counsel of Science and Technology (CONACyT) of Mexico as part of the project SEP-2011 Ciencia Basica 169765; PROMEP 103.5/11/4330; PAICYT 464-10. 


\section{References}

1. Cox, J.C., Shachat, J., Walker, M.: An experiment to evaluate Bayesian learning of Nash equilibrium plays. Games and Economic Behavior 34, 11-33 (2001)

2. Harsanyi, J.C.: Games with incomplete information played by 'Bayesian' players I-III. Management Sci. 14, 159-182, 320-334, 486-502 (1967)

3. Harsanyi, J.C., Selten, R.: A generalized Nash solution for two-person bargaining games with incomplete information. Management Sci. 18, 80-106 (1968)

4. Knight, F.H.: Risk, Uncertainty and Profit. The Riverside Press, Cambridge (1921)

5. Mertens, J.F., Zamir, S.: Formulation of Bayesian analysis for games with incomplete information. Int. J. Game Theory 14, 1-29 (1985)

6. Nash, J.F.: The bargaining problem. Econometrica 14, 155-162 (1950)

7. Osborne, M.J., Rubinstein, A.: Bargaining and Markets. Academic Press, New York (1990)

8. Osborne, M.J., Rubinstein, A.: A Course in Game Theory. MIT Press, Cambridge (1994)

9. Rubinstein, A.: "Afterword" of the 60th Anniversary Edition of J. Von Neumann O. Morgenstern's. In: Theory of Games and Economic Behavior, PDF File

10. Smith, V.L.: Bargaining and Market Behavior: Essays in Experimental Economics. Cambridge University Press, New York (2000)

11. Szidarovszky, F., Lin, C.H.: The alternating offer bargaining method under uncertainty. Applied Math. Comp. 76, 133-141 (1996) 